Journal of Algebraic Combinatorics, 21, 5-22, 2005 (c) 2005 Springer Science + Business Media, Inc. Manufactured in The Netherlands.

\title{
On Arc-Regular Permutation Groups Using Latin Squares
}

SÒNIA P. MANSILLA*

sonia@mat.upc.es

Escola Politècnica Superior de Castelldefels, Universitat Politècnica de Catalunya, Av. del Canal Olímpic, s/n, 08860 Castelldefels, Spain

Received March 2, 2001; Revised November 4, 2003; Accepted January 27, 2004

\begin{abstract}
For a given a permutation group $G$, the problem of determining which regular digraphs admit $G$ as an arc-regular group of automorphism is considered. Groups which admit such a representation can be characterized in terms of generating sets satisfying certain properties, and a procedure to manufacture such groups is presented. The technique is based on constructing appropriate factorizations of (smaller) regular line digraphs by means of Latin squares. Using this approach, all possible representations of transitive groups of degree up to seven as arc-regular groups of digraphs of some degree is presented.
\end{abstract}

Keywords: Cayley digraph, arc-transitive digraph, Latin square

\section{Introduction}

We are concerned only with directed graphs, called digraphs for short. A digraph $\Gamma=$ $(V, A)$ consists of a non-empty set $V=V(\Gamma)$ of vertices and a subset $A=A(\Gamma)$ of ordered pairs from $V$, called arcs. If $(u, v) \in A$ is an arc from $u$ to $v$ we say that $u$ is adjacent to $v$ and also that $v$ is adjacent from $u$. The sets of vertices adjacent to and adjacent from a vertex $v$ are denoted by $\Gamma^{-}(v)$ and $\Gamma^{+}(v)$, respectively. A digraph is regular of degree $r$ or $r$-regular when each vertex is adjacent to and from exactly $r$ vertices. A digraph $\Gamma$ is strongly connected whenever for any ordered pair of vertices $u, v \in V(\Gamma)$ there is a directed path from $u$ to $v$. We allow digraphs to have loops, that is, $\operatorname{arcs}(u, v)$ where $u=v$. A digraph with multiple arcs is known as multidigraph. For simplicity we sometimes speak of digraphs even if we actually allow multidigraphs. The order of a digraph is the cardinality of its set of vertices. A digraph is finite if its order is finite. Only finite, regular and strongly connected digraphs are considered in this paper. For further graph- and group-theoretic concepts not defined here we refer the reader to $[7,8]$.

Let $\Gamma=(V, A)$ be a digraph and let Aut $\Gamma$ denote its full automorphism group. If a subgroup $G$ of Aut $\Gamma$ acts transitively on the vertex set $V$ or the $\operatorname{arc}$ set $A$, we say that $\Gamma$ is $G$-vertex-transitive or $G$-arc-transitive, respectively. If $G$ acts regularly on $V$ or $A$, we say that $\Gamma$ is $G$-vertex-regular or $G$-arc-regular, respectively. We often omit the prefix ' $G^{\prime}$

*Partially supported by the Comissionat per a Universitats i Recerca of the Generalitat de Catalunya under Grant 1997FI-693, and through a European Community Marie Curie Fellowship under contract HPMF-CT-2001-01211. 
when $G=$ Aut $\Gamma$ and we simply say that $\Gamma$ is vertex-transitive $(V T)$, arc-transitive $(A T)$, vertex-regular or arc-regular.

Petersen [14] first asked which permutation groups are automorphism groups of some graph. Much later several more specific problems emerged. For instance, one was to characterize groups which have a graphical regular representation (GRR), that is, the problem was to find those groups $G$ for which there exists a graph $\Gamma$ with Aut $\Gamma \cong G$ acting regularly on $V(\Gamma)[1]$. The analogous version for digraphs was to characterize groups admitting a digraphical regular representation (DRR) [1].

We here consider a similar problem, namely, the following. A transitive permutation group $G$ on a set $\Omega$ is called arc-regular if there exists a regular digraph $\Gamma, V(\Gamma)=\Omega$, with $G \leq$ Aut $\Gamma$ as a group of automorphisms acting regularly on the set of its arcs. The problem is to characterize arc-regular groups. (The version involving sharply arc-transitive groups has been addressed in the literature by Babai et al. [2] and by Cameron [5].) The above problem (if we require that the digraphs in question are regular) is solved by our Theorem 9 and involves the concept of discordant permutations. (Two permutations are discordant if no symbol has the same image under both permutations.) More precisely, we prove that if $G$ is a permutation group which acts transitively on a set $\Omega$ of $n$ elements, then there exists a regular digraph of degree $r$ and order $n / r$ which is $G$-arc-regular if and only if $G$ is generated by a subset $F \subseteq G$ of $r$ pairwise discordant permutations of $\Omega$, such that the set $F F^{-1}$ is a subgroup of $G$ of order $r$. In this case, the set $F$ is a right coset of $F F^{-1}$ in $G$.

In [12] we introduced a construction of arc-transitive digraphs. Given an arbitrary regular digraph $\Gamma$ (possibly with loops), the construction provides instances of arc-transitive digraphs which cover the original digraph. In fact, the construction is also a construction of arc-regular permutation groups. If $\mathrm{L} \Gamma$ is the line digraph of the original digraph $\Gamma$, then the resulting arc-transitive digraphs are $G$-arc-regular provided that $G$ is the permutation group of an 'appropriate' factorization $F$ of L $\Gamma$. Such an 'appropriate' factorization $F$ of $\mathrm{L} \Gamma$ is called uniform. A factorization $F$ of a regular line digraph $\mathrm{L} \Gamma$ is uniform if and only if the corresponding Cayley cover $\overline{\mathrm{L}}_{F}$ is a line digraph (see Section 2 for definitions.)

It further transpires that uniform factorizations of regular line digraphs are closely related to uniform Latin squares (which are composition tables for groups, see Section 4). In fact, one can manufacturate all uniform factorizations of regular line digraphs (and consequently, digraphs admitting an arc-regular group of automorphisms) by means of such Latin squares. This is the essence of our new procedure to determine all arc-regular permutation groups. The procedure is illustrated by considering all digraphs of small order. Moreover, in Theorem 8 we enumerate uniform factorizations of a $r$-regular line digraph in terms of the number of normalized uniform Latin squares of order $r$. We also determine the number of uniform Latin squares of order $r$ for $r \leq 6$. In Corollary 6 , we give a characterization of uniform Latin squares which states that a Latin square is uniform if and only if the complete set of discordant permutations with which it is associated is a subgroup of $\mathcal{S}_{r}$.

The paper is organized as follows. In Section 2 we give the terminology and preliminary results to be used in the paper. In Section 3 we introduce uniform factorizations of line digraphs. In Section 4 we characterize uniform Latin squares and we give the construction 
of arc-regular permutation groups from an arbitrary regular digraph. The last Sections apply the construction to digraphs and transitive groups of small degree.

\section{Basic terminology}

\subsection{Digraphs}

Let us recall the definition of line digraph and some basic results. See [11] for more information or proofs on line digraphs not given here. The line digraph $\mathrm{L} \Gamma=\left(V_{\mathrm{L}}, A_{\mathrm{L}}\right)$ of $\Gamma=(V, A)$ has the arcs of $\Gamma$ as vertices and $\left(\left(x_{1}, x_{2}\right),\left(y_{1}, y_{2}\right)\right)$ is an arc in $\mathrm{L} \Gamma$ whenever $x_{2}=y_{1}$. Heuchenne's characterization of line digraphs states that a regular digraph $\Gamma=(V, A)$ is a line digraph of some (multi)digraph if and only if $\left\{\Gamma^{-} \Gamma^{+}(u): u \in V\right\}$ is a partition of the vertex set $V$. If $\Gamma=(V, A)$ is a regular digraph, then the map $\phi:$ Aut $\Gamma \rightarrow$ Aut L $\Gamma$ defined as

$$
(x, y)^{g^{\phi}}=\left(x^{g}, y^{g}\right),(x, y) \in A
$$

for each $g \in$ Aut $\Gamma$, is a group isomorphism. (Note that we use exponential notation for the action of permutation and mappings.) We identify $g^{\phi} \in$ Aut $L \Gamma$ with $g \in$ Aut $\Gamma$. With this identification, we can write Aut $\mathrm{L} \Gamma=$ Aut $\Gamma$. See figure 2 for an illustration of the complete digraph with loops $K_{2}^{+}$and its corresponding line digraph $\mathrm{L} K_{2}^{+}$.

We recall here the concept of factorization of a regular digraph. By the König-Hall theorem, a $r$-regular digraph $\Gamma=(V, A)$ is the sum of permutation digraphs $F=\left\{F_{1}, \ldots, F_{r}\right\}$ corresponding to a set $\left\{f_{1}, \ldots, f_{r}\right\}$ of permutations. We call such a set $F$ a factorization or an arc-coloring of $\Gamma$. That is, a factorization is a set of 1-regular spanning subdigraphs of $\Gamma$ whose sets of arcs partition $A$. Each $F_{i}$ is a disjoint union of directed cycles, and we interpret $f_{i}$ as the corresponding permutation of the vertex set $V$ whose disjoint cycle decomposition is $F_{i}, 1 \leq i \leq r$. We still denote by $F=\left\{f_{1}, \ldots, f_{r}\right\}$ the set of these permutations. Such a set $F$ generates a permutation group $G(\Gamma, F)$ on $V$, called the permutation group of the factorization $F$. (See in figure 2 a factorization $F_{B}$ of $\mathrm{LK}_{2}^{+}$whose permutation group is the alternating group $\mathcal{A}_{4}$.) An arc-colored digraph $(\Gamma, F)$ is a digraph $\Gamma$ together with a factorization $F$ of $\Gamma$. Two colored digraphs $(\Gamma, F)$ and $\left(\Gamma^{\prime}, F^{\prime}\right)$ are said to be isomorphic if there exist a digraph isomorphism $\Phi: \Gamma \rightarrow \Gamma^{\prime}$ and a bijection $\sigma: F \rightarrow F^{\prime}$ such that $\left(u^{f}\right)^{\Phi}=\left(u^{\Phi}\right)^{f^{\sigma}}$ for all $f \in F$ and $u \in V(\Gamma)$. We also say that $\Phi$ is a colored isomorphism. Similarly, an automorphism $\Phi$ of a digraph $\Gamma$ is said to be a colored automorphism of $(\Gamma, F)$ if there exists a permutation $\sigma$ on $F$ such that $\left(u^{f}\right)^{\Phi}=\left(u^{\Phi}\right)^{f^{\sigma}}$ for all $f \in F$ and $u \in V(\Gamma)$. If $\sigma$ is the identity, $\Phi$ is said to be strictly colored. The colored group $\operatorname{Aut}(\Gamma, F)$ of $(\Gamma, F)$ is the group of its colored automorphisms, and the $s$-colored group $\operatorname{Aut}^{\mathcal{S}}(\Gamma, F)$ of $(\Gamma, F)$ is the group of its strictly colored automorphisms. Note that $\operatorname{Aut}^{\mathcal{S}}(\Gamma, F)$ acts semiregularly on vertices because the digraphs are assumed connected, and it is not difficult to check that $\operatorname{Aut}^{\mathcal{S}}(\Gamma, F)$ is a normal subgroup of $\operatorname{Aut}(\Gamma, F)$.

A semicomplete bipartite digraph has a vertex set consisting of two disjoint sets $V_{0}$ and $V_{1}$ of equal cardinality (called stable sets) and all possible arcs from $V_{0}$ to $V_{1}$. If $\left|V_{0}\right|=\left|V_{1}\right|=r$ we denote such a digraph by $K_{r, r}$. A factorization (or coloring) of $K_{r, r}$ is 
a partition of its arc set into $r$ subsets such that each subset consists of $r$ disjoint arcs. This is equivalent to specifying a set of bijections $F=\left\{f_{1}, \ldots, f_{r}\right\}$ from $V_{0}$ to $V_{1}$. We define a colored isomorphism of two colored semicomplete bipartite digraphs similarly as in the case of regular digraphs (details are left to the reader). Note that all facts about the group of strictly colored automorphisms remain valid. A colored isomorphism $(\Gamma, F) \rightarrow\left(\Gamma^{\prime}, F^{\prime}\right)$ of two semicomplete bipartite digraphs together with fixed chosen bijections $F \rightarrow \mathcal{C}$ and $F^{\prime} \rightarrow \mathcal{C}$, is a color preserving isomorphism if it induces the identity mapping on $\mathcal{C}$ (via the bijections onto $\mathcal{C}$ ). Obviously, a color preserving isomorphism is uniquely determined by the image on one vertex, and color preserving automorphism coincide with the strictly colored ones.

A surjective digraph homomorphism $\sigma: \Gamma_{1} \rightarrow \Gamma_{2}$ is called a covering map if $\left|v^{\sigma^{-1}}\right|$ does not depend on $v \in V\left(\Gamma_{2}\right)$ and if $\left|\Gamma_{1}^{+}(u)\right|=\left|\Gamma_{2}^{+}\left(u^{\sigma}\right)\right|$ holds for all $u \in V\left(\Gamma_{1}\right)$ (that is, if the outcomming arcs of a vertex are bijectively mapped to the outcomming arcs of the image of that vertex.) If $\sigma: \Gamma_{1} \rightarrow \Gamma_{2}$ is a covering map we call $\Gamma_{1}$ a cover of $\Gamma_{2}$. For instance, the digraph homomorphism $\sigma: V(\mathrm{~L} \Gamma) \rightarrow V(\Gamma)$ defined by $(u, v)^{\sigma}=v$ is the so called standard covering map from $\mathrm{L} \Gamma$ onto $\Gamma$. The reader is warned that there are several definitions of the concept of cover of digraph in the literature.

Let $G$ be a finite group and $S \subset G$. The Cayley digraph of $G$ relative to $S, \operatorname{Cay}(G, S)$, has the elements of $G$ as vertices and there is an $\operatorname{arc}(x, x s)$ for each $x \in G$ and each $s \in S$. Let $\Gamma$ be a connected regular digraph and $G=G(\Gamma, F)$ the permutation group of a factorization $F$ of $\Gamma$. It is not difficult to check that $\bar{\Gamma}_{F}=\operatorname{Cay}(G, F)$ is a cover of $\Gamma$, called the Cayley cover of $\Gamma$ relative to $F$. See figure 2 for an illustration of a Cayley cover of $\mathrm{LK}_{2}^{+}$relative to the factorization shown in the figure.

\subsection{Latin squares}

Referring to Latin squares we use here the notation introduced in [15]. A Latin square of order $r$ is an ordered 4-tuple $(R, C, S ; L)$, where $R, C$ and $S$ are sets with $r$ elements and $L: R \times C \rightarrow S$ is a mapping with the following property: for each pair $(j, s) \in C \times S$ there is a unique $i \in R$ such that $L(i, j)=s$, and for each $(i, s) \in R \times S$ there is a unique $j \in C$ such that $L(i, j)=s$. The elements of $R, C, S$ of a Latin square $(R, C, S ; L)$ are usually called rows, columns, and symbols of the Latin square. We usually represent a Latin square $(R, C, S ; L)$ as an $r \times r$ matrix where the entry $(i, j)$ is the symbol $L(i, j)$. (Note that exponential notation is not used for the mapping $L$.)

Let $(R, C, S ; L)$ be a Latin square of order $r$. Let $Q$ be a set with $r$ elements. Any ordered triple $\pi_{R}, \pi_{C}, \pi_{S}$ of bijections from $R, C$ and $S$ to $Q$, respectively, induces a binary operation $\oplus$ on $Q$ given by

$$
q_{1} \oplus q_{2}=L\left(q_{1}^{\pi_{R}^{-1}}, q_{2}^{\pi_{C}^{-1}}\right)^{\pi_{S}}, \quad \text { for } q_{1}, q_{2} \in Q
$$

The definition of a Latin square ensures that $(Q, \oplus)$ is a quasigroup. We say that $(Q, \oplus)$ is a quasigroup associated with $(R, C, S ; L)$. In general, different choices of bijections $\pi_{R}, \pi_{C}, \pi_{S}$ give rise to different quasigroups. We say that the Latin square $(R, C, S ; L)$ is a composition table for a group $(H, \cdot)$ if one quasigroup $(Q, \oplus)$ associated with $(R, C, S ; L)$ 
is isomorphic to $(H, \cdot)$ (and hence every quasigroup with identity with which it is associated is isomorphic to $(H, \cdot))$. For more details see [3].

Two Latin squares $(R, C, S ; L)$ and $\left(R^{\prime}, C^{\prime}, S^{\prime} ; L^{\prime}\right)$ are said to be isomorphic (or equivalent) if there exist bijections $\sigma: R \rightarrow R^{\prime}, \tau: C \rightarrow C^{\prime}$ and $\pi: S \rightarrow S^{\prime}$ such that

$$
L^{\prime}(i, j)=L\left(i^{\sigma^{-1}}, j^{\tau^{-1}}\right)^{\pi}
$$

for all $(i, j) \in R^{\prime} \times C^{\prime}$. In particular, they are called strictly isomorphic whenever $S=S^{\prime}$ and $\pi$ is the identity. Clearly isomorphism and strictly isomorphism are equivalence relations. Moreover, if $(R, C, S ; L)$ is a Latin square and $\sigma: R \rightarrow R^{\prime}, \tau: C \rightarrow C^{\prime}$ and $\pi: S \rightarrow S^{\prime}$ are arbitrary bijections, then $\left(R^{\prime}, C^{\prime}, S^{\prime} ; L^{\prime}\right)$ with $L^{\prime}$ defined by the above equality, is an equivalent Latin square. Thus, each equivalence class of Latin squares has a representative in which $R=C=S=\{1,2, \ldots, r\}$ and $L(1, j)=L(j, 1)=j$ for $1 \leq j \leq r$. Such a Latin square is called normalized.

An automorphism of a Latin square $(R, C, S ; L)$ is an ordered triple $(\sigma, \tau, \pi)$ of bijections $\sigma: R \rightarrow R, \tau: C \rightarrow C$ and $\pi: S \rightarrow S$ such that $L\left(i^{\sigma}, j^{\tau}\right)=L(i, j)^{\pi}$. The collection of all automorphisms, with componentwise composition, constitutes the automorphism group $\operatorname{Aut}(R, C, S ; L)$. An automorphism of the form $(\sigma, \tau, \mathrm{id})$ is called strict, or an $s$ automorphism. The collection of all strict automorphisms constitutes a normal subgroup $\operatorname{Aut}^{\mathcal{S}}(R, C, S ; L)$ of $\operatorname{Aut}(R, C, S ; L)$.

Note that both automorphism groups $\operatorname{Aut}(R, C, S ; L)$ and $\operatorname{Aut}^{\mathcal{S}}(R, C, S ; L)$ of a Latin square $(R, C, S ; L)$ act in a natural way on the set of columns $C$ and on the set rows $R$. Furthermore, the group $\operatorname{Aut}^{\mathcal{S}}(R, C, S ; L)$ is semiregular on $R$ and or $C$. We say that a Latin square $(R, C, S ; L)$ is uniform if $\operatorname{Aut}^{\mathcal{S}}(R, C, S ; L)$ acts regularly on $R$. In fact, note that $\operatorname{Aut}^{\mathcal{S}}(R, C, S ; L)$ acts regularly on $R$ if and only if it acts regularly on $C$.

\section{Uniform factorizations of line digraphs}

Let $\Gamma_{0}$ be a connected regular digraph (possibly with loops). A factorization $F$ of the line digraph $\Gamma=\mathrm{L} \Gamma_{0}$ such that the resulting Cayley cover $\bar{\Gamma}_{F}$ is also a line digraph is said to be a uniform factorization of $\Gamma$. The construction of arc-transitive covers introduced in [12] was based on the fact that, if $\bar{\Gamma}_{F}=\mathrm{L} \Gamma^{\prime}$, then $\Gamma^{\prime}$ is an arc-transitive (multi)digraph and it is also a covering digraph of $\Gamma_{0}[12$, Theorem 1]. The diagram in figure 1 illustrates the construction.

In figure 2 we illustrate the construction of an arc-transitive cover of the complete digraph with loops $K_{2}^{+}$. We assign to the line digraph of $K_{2}^{+}$the uniform factorization $F_{B}$ shown in the figure, and consequently, the Cayley cover Cay $\left(\mathrm{L} K_{2}^{+}, F_{B}\right)$ is a line digraph of an

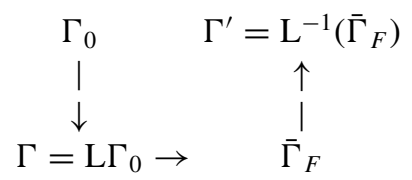

Figure 1. Diagram of the construction. 

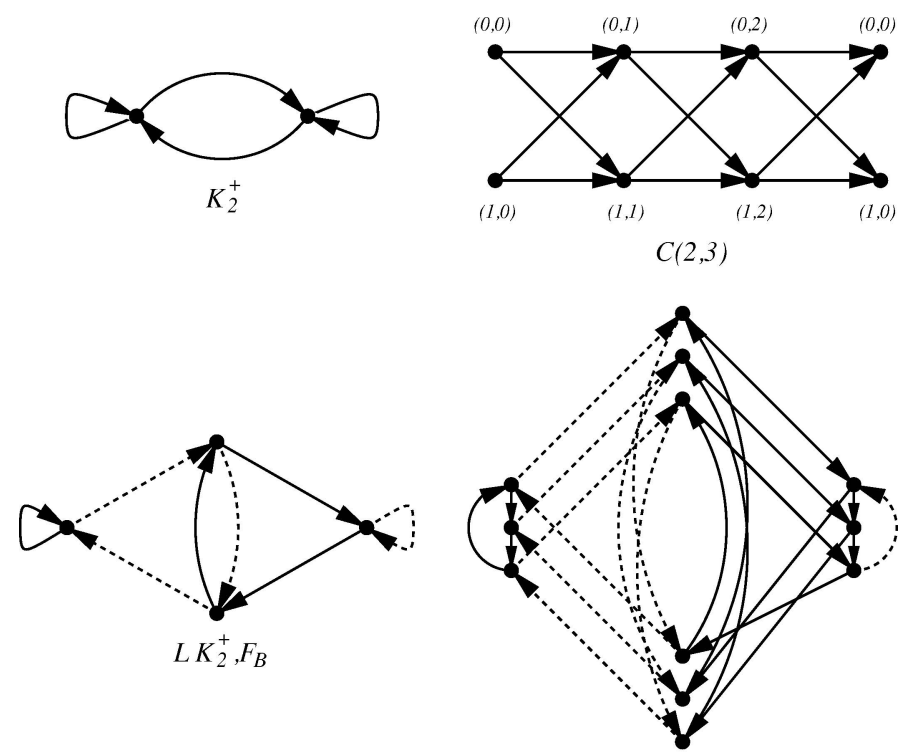

$\operatorname{Cay}\left(A_{4}, F_{B}\right)$

Figure 2. Construction of an arc-transitive cover of $K_{2}^{+}$.

arc-transitive digraph. In this case, the resulting arc-transitive cover of $K_{2}^{+}$is the complete generalized cycle $C(2,3)=\operatorname{Cay}\left(\mathbb{Z}_{2} \times \mathbb{Z}_{3},\left\{(i, 1): i \in \mathbb{Z}_{2}\right\}\right)$. And in particular, we find that $C(2,3)$ is an $\mathcal{A}_{4}$-arc-regular digraph.

A characterization of uniform factorizations which will be useful in the last section is the following.

Theorem 1 Let $F$ be a factorization of a $r$-regular connected line digraph $\Gamma$ and let $G=G(\Gamma, F)$ be the permutation group of $F$. Then, $F$ is uniform if and only if the set $F F^{-1}$ is a subgroup of $G$ of order $r$.

Proof: Suppose that $F$ is uniform. Then, $\bar{\Gamma}_{F}=\mathrm{L} \Gamma_{0}$ for some (multi)digraph $\Gamma_{0}$. For $f \in F$ consider the set $H=f F^{-1}$. For all $g \in F$, we have

$$
1 \in f F^{-1} \cap g F^{-1}=\bar{\Gamma}_{F}^{-1}(f) \cap \bar{\Gamma}_{F}^{-1}(g) .
$$

Then, Heuchenne's characterization of line digraphs implies $H=f F^{-1}=g F^{-1}=$ $F F^{-1}$ and $|H|=r$. In particular, $H=F f^{-1}$. Therefore, $H^{2}=\left(F f^{-1}\right)\left(f F^{-1}\right)=H$, and $H$ is a subgroup of $G$.

Conversely, suppose that $H=F F^{-1}$ is a subgroup of $G$ of order $r$. In particular, we have $H=f F^{-1}$ for any $f \in F$. Now, the set $\left\{\bar{\Gamma}_{F}^{-1}(g): g \in G\right\}$ is the partition of $G$ in left 
cosets of $H$ in $G$, since

$$
g F^{-1}=g f^{-1} f F^{-1}=g f^{-1} H .
$$

Hence, again by Heuchenne's characterization of line digraphs, the Cayley cover $\bar{\Gamma}_{F}$ is a line digraph and so, $F$ is uniform.

There is another characterization of uniform factorizations of regular line digraphs proved in [12]. Let $\Gamma$ be a regular line digraph of degree $r$ and $F$ a factorization of $\Gamma$. For each $u \in V(\Gamma)$, let $\left(\Gamma^{u}, F_{u}\right)$ denote the bipartite arc-colored digraph with stable sets $V_{0}^{u}=$ $\Gamma^{-} \Gamma^{+}(u) \times\{0\}$ and $V_{1}^{u}=\Gamma^{+}(u) \times\{1\}$, and with an $\operatorname{arc}((x, 0),(y, 1))$ in $\Gamma^{u}$ whenever $(x, y)$ is an arc of $\Gamma$. (Note that this forces $\Gamma^{u}$ to be bipartite, even in the case where $\Gamma$ has loops and $\Gamma^{-} \Gamma^{+}(u) \cap \Gamma^{+}(u) \neq \emptyset$.) The digraph $\Gamma^{u}$ is isomorphic to $K_{r, r}$. Moreover, an arc $((x, 0),(y, 1))$ in $\Gamma^{u}$ is colored with the permutation $f \in F$ such that $y=x^{f}$. With these definitions and remarks, the following holds.

Theorem 2 ([12]). Let $F$ be a factorization of a regular connected line digraph $\Gamma$. Then $F$ is uniform if and only if for each pair of vertices $u, v \in V(\Gamma)$ there is a color preserving digraph isomorphism $\phi_{u, v}:\left(\Gamma^{u}, F_{u}\right) \rightarrow\left(\Gamma^{v}, F_{v}\right)$ such that $(u, 0)^{\phi_{u, v}}=(v, 0)$.

A factorization of a semicomplete bipartite digraph is called uniform if the corresponding group of strictly colored automorphisms acts transitively (and hence regularly) on each of the stable sets of vertices. If $F$ is a uniform factorization of a regular line digraph $\Gamma$ it follows from the above characterization that for each $u \in V(\Gamma)$ the resulting factorization $F_{u}$ of $\Gamma^{u}$ is uniform. Moreover, $F$ can be recaptured from a 'basic set of these factorizations' as follows.

Let $\mathcal{R}=\left\{u_{1}, \ldots, u_{s}\right\}$ be a minimal set of vertices of a $r$-regular line digraph $\Gamma$ with respect to the property that $V=\cup_{u_{i} \in \mathcal{R}} \Gamma^{-} \Gamma^{+}\left(u_{i}\right)$ (from Heuchenne's characterization, $s=\frac{|V|}{r}$.) Then, the set $\left(\Gamma^{u_{1}}, F_{1}\right), \ldots,\left(\Gamma^{u_{s}}, F_{s}\right)$, where $F_{i}=\left\{f_{i 1}, \ldots, f_{i r}\right\}$ is an arbitrary factorization of $\Gamma^{u_{i}}$, induces a factorization $F$ of $\Gamma$. Namely, for each $\operatorname{arc}(u, v) \in A$, there exists a unique $u_{i} \in \mathcal{R}$ such that $u \in \Gamma^{-} \Gamma^{+}\left(u_{i}\right)$ and we can assign to the arc $(u, v)$ the color $f_{j}$ if $(u, 0)^{f_{i j}}=(v, 1)$.

The next result follows immediately from the discussion above.

Corollary 3 Let $F$ be a factorization of a regular line digraph $\Gamma$ generated by arbitrary factorizations $\left(\Gamma^{u_{i}}, F_{i}\right), i=1, \ldots, s$, as explained above. Then $F$ is uniform if and only if - for each $i=2, \ldots, s$, there is a color preserving isomorphism $\left(\Gamma^{u_{1}}, F_{1}\right) \rightarrow\left(\Gamma^{u_{i}}, F_{i}\right)$ mapping $\left(u_{1}, 0\right)$ to $\left(u_{i}, 0\right)$, and

- the factorization of $\left(\Gamma^{u_{1}}, F_{1}\right)$ is uniform.

This corollary gives a procedure how to generate uniform factorizations of $\Gamma$ provided that we are given some uniform factorization $F_{1}$ of $\Gamma^{u_{1}}$ : one only needs to transfer consistently the coloring $F_{1}$ by an isomorphism $\left(\Gamma^{u_{1}}, F_{1}\right) \rightarrow\left(\Gamma^{u_{i}}, F_{i}\right)$ mapping $\left(u_{1}, 0\right) \mapsto\left(u_{i}, 0\right)$ to a coloring $F_{i}$ of $\Gamma^{u_{i}}$. We shall return to this in the next section. 
Note that we can identify each of the arc-colored digraphs $\left(\Gamma^{u}, F_{u}\right)$ with a Latin square $\left(V_{0}^{u}, V_{1}^{u}, F_{u} ; L\right)$ where

$$
L: V_{0}^{u} \times V_{1}^{u} \rightarrow F_{u}
$$

is the mapping defined by $L((x, 0),(y, 1))=f$ whenever $(x, 0)^{f}=(y, 1)$. We say that the Latin square $\left(V_{0}^{u}, V_{1}^{u}, F_{u} ; L\right)$ is associated with $\left(\Gamma^{u}, F_{u}\right)$. Note that the color preserving isomorphisms $\left(\Gamma^{u}, F_{u}\right) \rightarrow\left(\Gamma^{v}, F_{v}\right)$ of Theorem 2 correspond to strict isomorphisms of associated Latin squares, and hence strictly colored automorphisms of $\left(\Gamma^{u}, F_{u}\right)$ correspond to strict automorphisms of the associated Latin square. We prove this last assertion formally below.

Theorem 4 Let $\Gamma=(V, A)$ be a regular line digraph and $u \in V$. Let $F_{u}$ be a factorization of $\Gamma^{u}$, and let $\left(V_{0}^{u}, V_{1}^{u}, F_{u} ; L\right)$ be the Latin square associated with the arc-colored digraph $\left(\Gamma^{u}, F_{u}\right)$.

Then, $F_{u}$ is a uniform factorization of $\Gamma^{u}$ if and only $\left(V_{0}^{u}, V_{1}^{u}, F_{u} ; L\right)$ is uniform.

Proof: Recall that if $K_{r, r}$ is a semicomplete bipartite digraph with stable sets $V_{0}^{u}$ and $V_{1}^{u}$, then Aut $K_{r, r}=\mathcal{S}_{r} \times \mathcal{S}_{r}$, where $(\sigma, \tau) \in \mathcal{S}_{r} \times \mathcal{S}_{r}$ acts on $V_{0}^{u} \sqcup V_{1}^{u}$ as $x^{\sigma}$ if $x \in V_{0}^{u}$, and as $y^{\tau}$ if $y \in V_{1}^{u}$. Thus, we can consider the mapping

$$
\Phi: \operatorname{Aut}^{\mathcal{S}}\left(\Gamma^{u}, F_{u}\right) \rightarrow \operatorname{Aut}^{\mathcal{S}}\left(V_{0}^{u}, V_{1}^{u}, F_{u} ; L\right)
$$

defined by $\phi \mapsto\left(\sigma, \tau\right.$, id), where $x^{\phi}=x^{\sigma}$ for $x \in V_{0}^{u}$, and $y^{\phi}=y^{\tau}$ for $y \in V_{1}^{u}$.

Clearly, $\Phi$ is a bijection and it is easy to check that it is a group homomorphism as well. Furthermore, the actions of $\operatorname{Aut}^{\mathcal{S}}\left(\Gamma^{u}, F_{u}\right)$ and $\operatorname{Aut}^{\mathcal{S}}\left(V_{0}^{u}, V_{1}^{u}, F_{u} ; L\right)$ on $V_{0}^{u}$ are permutationally equivalent. Finally, via the isomorphism $\Phi$, the group $\operatorname{Aut}^{\mathcal{S}}\left(\Gamma^{u}, F_{u}\right)$ is regular on $V_{0}^{u}$ if and only if $\operatorname{Aut}^{\mathcal{S}}\left(V_{0}^{u}, V_{1}^{u}, F_{u} ; L\right)$ is regular on the set of rows $V_{0}^{u}$.

\section{Uniform Latin squares and uniform factorization of line digraphs}

In this section we characterize uniform Latin squares in terms of discordant permutations, and we give a procedure for manufacturing arc-regular permutation groups from an arbitrary regular digraph, using uniform Latin squares.

We say that two permutations are discordant if no symbol has the same image under both permutations. We say that a set of (pairwise) discordant permutations of degree $r$ is a complete set if it has cardinality $r$, and so acts as a sharply transitive set of permutations on the symbols. Cayley [6] introduced a one to one correspondence between Latin squares of order $r$ and complete sets of discordant permutations of degree $r$ in the following way. We identify each row $i \in R$ of a normalized Latin square $(R, C, S ; L)$ of order $r$ with the permutation $\delta_{i} \in \mathcal{S}_{r}$ such that $j^{\delta_{i}}=L(i, j)$ for each $j \in C$. It is easy to check that the resulting set of permutations $\left\{\delta_{1}, \ldots, \delta_{r}\right\}$ is a complete set of discordant permutations. For instance, the Latin square in figure 3 corresponds to the following complete set of discordant 


$$
\left(\begin{array}{lllll}
1 & 2 & 3 & 4 & 5 \\
2 & 1 & 4 & 5 & 3 \\
3 & 4 & 5 & 2 & 1 \\
4 & 5 & 1 & 3 & 2 \\
5 & 3 & 2 & 1 & 4
\end{array}\right)
$$

Figure 3. A non uniform Latin square of order 5.

permutations:

$$
\{(),(1,2)(3,4,5),(1,3,5)(2,4),(1,4,3)(2,5),(1,5,4)(2,3)\} .
$$

Theorem 5 Let $(R, C, S ; L)$ be a normalized Latin square of order $r$. Then $(R, C, S ; L)$ is uniform if and only if the complete set of discordant permutations corresponding to $(R, C, S ; L)$ is a subgroup of $\mathcal{S}_{r}$ permutationally equivalent to the action of $\operatorname{Aut}^{\mathcal{S}}(R, C, S ; L)$ on $C$.

Proof: If $(R, C, S ; L)$ is uniform, then $\operatorname{Aut}^{\mathcal{S}}(R, C, S ; L)$ acts regularly on $C$ (and $R$.) Let us consider

$$
\operatorname{Aut}^{\mathcal{S}}(R, C, S ; L)=\left\{\left(\sigma_{1}, \tau_{1}, \mathrm{id}\right), \ldots,\left(\sigma_{r}, \tau_{r}, \mathrm{id}\right)\right\}
$$

where the automorphisms are indexed in such a way that $i^{\sigma_{i}}=1$.

Then, for each $r_{0} \in R$, the bijection $\tau_{r_{0}}$ is determined by

$$
L\left(r_{0}, i\right)=L\left(r_{0}^{\sigma_{r_{0}}}, i^{\tau_{r_{0}}}\right)=L\left(1, i^{\tau_{r_{0}}}\right)=i^{\tau_{r_{0}}}
$$

and therefore $\tau_{r_{0}}$ is just the row $r_{0}$ regarded as a permutation of $\mathcal{S}_{r}$.

Conversely, if the complete set of discordant permutations corresponding to $(R, C, S ; L)$ is a subgroup of $\mathcal{S}_{r}$, then it is a subgroup that acts regularly on $\{1, \ldots, r\}$. Thus, Aut $\mathcal{S}^{\mathcal{S}}$ $(R, C, S ; L)$ acts regularly on $C$ (and so on $R$ ), and $(R, C, S ; L)$ is uniform.

The above Theorem is a useful tool to determine if a normalized Latin square is uniform or not. For instance, by Theorem 5, the Latin square in figure 3 is not uniform, since the corresponding complete set of discordant permutations is not a subgroup of $\mathcal{S}_{r}$.

As a Corollary of Theorem 5 we characterize uniform Latin squares as Latin squares which are composition tables of groups.

Corollary 6 A normalized Latin square $\mathcal{Q}=(R, C, S ; L)$ of order $r$ is uniform if and only if it is a composition table for a group, say $(H, \cdot)$. In such a case, $H$ is isomorphic to the corresponding group of discordant permutations $\left\{\delta_{1}, \ldots, \delta_{r}\right\}$. 
Proof: Let us suppose that $\mathcal{Q}$ is a composition table for a group $(H, \cdot)$. Then there exist bijections $\pi_{R}, \pi_{C}, \pi_{S}$ from $R, C, S$ to the set of elements of the group $H$, such that the binary operation of $H$ is defined by

$$
h_{1} \cdot h_{2}=L\left(h_{1}^{\pi_{R}^{-1}}, h_{2}^{\pi_{C}^{-1}}\right)^{\pi_{S}}
$$

for any $h_{1}, h_{2} \in H$. For each permutation $\delta_{i}$ associated with $(R, C, S ; L)$, let $\gamma_{\pi_{R}(i)} \in$ $\operatorname{Sym}(H)$ be the permutation defined by $h^{\gamma_{\pi^{(i)}}}=\left(\left(h^{\pi_{C}^{-1}}\right)^{\delta_{i}}\right)^{\pi_{S}}$ for each $h \in H$. Then,

$$
h^{\gamma_{\pi^{(i)}}}=\left(\left(h^{\pi_{C}^{-1}}\right)^{\delta_{i}}\right)^{\pi_{S}}=L\left(i, h^{\pi_{C}^{-1}}\right)^{\pi_{S}}=i^{\pi_{R}} \cdot h .
$$

That is, the set of permutations $\left\{\gamma_{1}, \ldots, \gamma_{r}\right\}$ is the left representation by permutations of the group $H$. Then, since $\left\{\gamma_{1}, \ldots, \gamma_{r}\right\}$ is a subgroup of $\operatorname{Sym}(H)$ isomorphic to $H$, the complete set of discordant permutations $\left\{\delta_{1}, \ldots, \delta_{r}\right\}$ is a subgroup of $\mathcal{S}_{r}$ (isomorphic to $H$ ). By Theorem 5, $\mathcal{Q}$ is uniform.

Conversely, suppose that $\mathcal{Q}$ is a uniform Latin square. Let us write $H=\left\{\delta_{1}, \ldots, \delta_{r}\right\}$. By Theorem 5, $H<\mathcal{S}_{r}$. Note that $\delta_{i}$ verifies $1^{\delta_{i}}=i$, and then $\delta_{k} \circ \delta_{i}=\delta_{r}$ where $r=1^{\delta_{i} \delta_{k}}$.

Let $\pi_{R}, \pi_{C}, \pi_{S}:\{1, \ldots, r\} \rightarrow H$ be the bijections defined by $i^{\pi_{R}}=i^{\pi_{C}}=i^{\pi_{S}}=\delta_{i}$ for $i \in\{1, \ldots, r\}$. Then,

$$
\delta_{k} \circ \delta_{i}=L\left(\delta_{k}^{\pi_{R}^{-1}}, \delta_{i}^{\pi_{C}^{-1}}\right)^{\pi_{S}}=\delta_{L(k, i)}=\delta_{1 \delta_{i} \delta_{k}}
$$

Hence, $\mathcal{Q}$ is a composition table for $H$.

Note that two uniform Latin squares are isomorphic if and only if they are associated with isomorphic groups. Hence the number of isomorphism classes of uniform Latin squares of order $r$ is equal to the number of pairwise non isomorphic groups of order $r$. Each isomorphism class associated with a group, say $H$, splits into strict isomorphism classes, each being canonically represented by a unique normalized Latin square. The number of such squares is equal to the number of regular representations of $H$ as a subgroup of the symmetric group $\mathcal{S}_{r}$, that is, to the number of conjugates of $H$ within $\mathcal{S}_{r}$.

We next enumerate all uniform factorizations of a $r$-regular line digraph in terms of the number of normalized uniform Latin squares of order $r$. In the general case, it is proved in [4] that a $r$-regular line digraph of order $n$ admits $L(r)^{\frac{n}{r}}$ factorizations (up to permutations of colors), where $L(r)$ is the number of normalized Latin squares of order $r$. In the proof of Theorem 8 we (implicitly) give a a procedure to manufacture all uniform factorizations of a $r$-regular line digraph, provided that we know all the normalized uniform Latin squares of order $r$.

Recall from previous section how to manufacturate a uniform factorization of a $r$-regular line digraph from a given uniform factorization of $K_{r, r}$. A reformulation in terms of uniform Latin squares gives the following result.

Corollary 7 Let $\Gamma=(V, A)$ be a regular line digraph of order $n$ and degree $r$, and let $\mathcal{R}=\left\{u_{1}, \ldots, u_{n / r}\right\} \subseteq V$ such that $V=\bigcup_{u_{i} \in \mathcal{R}} \Gamma^{-} \Gamma^{+}\left(u_{i}\right)$. For $i=1, \ldots, n / r, \operatorname{let}\left(\Gamma^{u_{i}}, F_{i}\right)$ 
be an arbitrary factorization and denote by $\mathcal{Q}_{i}$ the normalized Latin square associated with it, respectively. Let $F$ be a factorization of $\Gamma$ generated by $\left(\Gamma^{u_{i}}, F_{i}\right), i=1, \ldots, n / r$.

Then $F$ is uniform if and only if

- for each $i=2, \ldots, n / r, \mathcal{Q}_{i}$ and $\mathcal{Q}_{1}$ are strictly isomorphic, and

- $\mathcal{Q}_{1}$ is uniform.

Proof: By Corollary 3, $F$ is uniform if $\left(\Gamma^{u_{1}}, F_{1}\right)$ is uniform and for each $i=2, \ldots, n / r$, there is a color preserving isomorphism $\left(\Gamma^{u_{1}}, F_{1}\right) \rightarrow\left(\Gamma^{u_{i}}, F_{i}\right)$ mapping $\left(u_{1}, 0\right)$ to $\left(u_{i}, 0\right)$. By Theorem 4, $\left(\Gamma^{u_{1}}, F_{1}\right)$ is uniform if and only if $\mathcal{Q}_{1}$ is uniform. The result follows from the remark that color preserving isomorphisms of semicomplete bipartite digraphs correspond to strict isomorphisms of the associated Latin squares.

Theorem 8 Let $\Gamma=(V, A)$ be a regular connected line digraph of order $n$ and degree $r$. Let $L_{r}$ be the number of normalized uniform Latin squares of order $r$.

Then, $\Gamma$ admits

$$
(r-1) !^{\frac{n}{r}} r !^{\frac{n}{r}-1} L_{r}
$$

different uniform factorizations (up to permutation of colors).

Proof: Let $\mathcal{R}=\left\{u_{1}, \ldots, u_{n / r}\right\}$ be a set of vertices of $\Gamma$ such that $V=\cup_{u_{i} \in \mathcal{R}} \Gamma^{-} \Gamma^{+}\left(u_{i}\right)$. Let us consider $u_{1} \in \mathcal{R}$ and $\Gamma^{+}\left(u_{1}\right)$. Since we are counting factorizations up to permutations of colors, we can fix the colors of the arcs from $u_{1}$ to vertices in $\Gamma^{+}\left(u_{1}\right)$ without loss of generality.

Let $\mathcal{Q}$ be a normalized uniform Latin square of order $r$. We first calculate the number of uniform factorizations of each $\Gamma^{u_{i}}$ with the associated normalized Latin squares isomorphic to $\mathcal{Q}$.

In the case of $\Gamma^{u_{1}}$, we have to determine the colors of all the arcs of $\Gamma^{u_{1}}$ with initial vertex in $V_{0} \backslash\left\{\left(u_{1}, 0\right)\right\}$. This is equivalent to assigning to each vertex in $V_{0} \backslash\left\{\left(u_{1}, 0\right)\right\}$ the number of a row of the Latin square $\mathcal{Q}$ (except the row of $\left.\left(u_{1}, 0\right)\right)$. There are $(r-1)$ ! choices.

In the case of $\Gamma^{u_{i}}$ for $i>1$, we can only fix the row of a vertex in the first stable set, for instance $\left(u_{i}, 0\right)$. Then, there are $(r-1)$ ! choices for assigning a row number to the vertices in $V_{0} \backslash\left\{\left(u_{i}, 0\right)\right\}$, and $r$ ! choices for assigning a column number to the vertices in $V_{1}$. In total, this gives $(r-1) ! r$ ! choices for positioning the elements of $V\left(\Gamma^{u_{i}}\right) \backslash\left\{\left(u_{i}, 0\right)\right\}$.

Hence, the number of uniform factorizations of $\Gamma$ arising from a given Latin square $\mathcal{Q}$ is

$$
(r-1) ! \prod_{i=2}^{n / r}((r-1) ! r !)=(r-1) !^{\frac{n}{r}} r !^{\frac{n}{r}-1}
$$

Finally, note that the actual choice of vertices in $\mathcal{R}$ was not relevant.

In particular, note that the number of uniform factorizations of a regular line digraph depends only on its degree of regularity and order. Thus, two regular line digraphs of the same order and degree admit the same number of uniform factorizations. 
Finally, we note that the automorphism group Aut $\Gamma$ of a digraph $\Gamma=(V, A)$ acts in a natural way on the set of factorizations of the digraph. Namely, for each $\sigma \in$ Aut $\Gamma$ and each factorization $(F, \phi)$ of $\Gamma$, we define $\left(F, \phi_{\sigma}\right)$ as the factorization of $\Gamma$ where $\phi_{\sigma}: A \rightarrow F$ is defined by $(u, v)^{\phi_{\sigma}}=\left(u^{\sigma^{-1}}, v^{\sigma^{-1}}\right)^{\phi}$, for $(u, v) \in A$.

Moreover, if $\Gamma$ is a line digraph this action of Aut $\Gamma$ on the set of factorizations of $\Gamma$ maps uniform factorizations to uniform factorizations. We say that two factorizations $F_{1}$ and $F_{2}$ of a digraph are equivalent if there exists an automorphism of the digraph which maps $F_{1}$ to $F_{2}$. In particular, if two factorizations of the digraph are equivalent, their associated Latin squares are isomorphic. Obviously, equivalent factorizations generate isomorphic permutation groups and furthermore, Cayley covers corresponding to equivalent factorizations are isomorphic.

\section{Uniform Latin squares of small order}

We illustrate the above results by constructing all uniform factorizations of line digraphs of digraphs of small degree.

Cases $r=2,3$. There exists a unique normalized Latin square of order 2 and a unique normalized Latin square of order 3, which are clearly uniform Latin squares. Since these Latin squares are unique, all factorizations of regular line digraphs of degree 2 or 3 are uniform. By Theorem 2, this is equivalent to saying that all Cayley covers of 2-regular or 3-regular line digraphs are also line digraphs. The 2-regular case was considered in [13].

Case $r=4$. There are four normalized Latin squares of order 4, namely ${ }^{1}$ :

$$
\left(\begin{array}{llll}
1 & 2 & 3 & 4 \\
2 & 3 & 4 & 1 \\
3 & 4 & 1 & 2 \\
4 & 1 & 2 & 3
\end{array}\right), \quad\left(\begin{array}{llll}
1 & 2 & 3 & 4 \\
2 & 4 & 1 & 3 \\
3 & 1 & 4 & 2 \\
4 & 3 & 2 & 1
\end{array}\right), \quad\left(\begin{array}{llll}
1 & 2 & 3 & 4 \\
2 & 1 & 4 & 3 \\
3 & 4 & 2 & 1 \\
4 & 3 & 1 & 2
\end{array}\right), \quad\left(\begin{array}{llll}
1 & 2 & 3 & 4 \\
2 & 1 & 4 & 3 \\
3 & 4 & 1 & 2 \\
4 & 3 & 2 & 1
\end{array}\right)
$$

It is easy to check that the first three represent a composition table for the cyclic group $\mathbb{Z}_{4}$ and that the last one represents a composition table for the Klein group $V_{4}=\mathbb{Z}_{2}^{2}$. Therefore, the four of them are uniform.

Let us apply this result to the complete digraph with loops $K_{4}^{+}$, which is a line digraph of a multidigraph with a unique vertex and four multiple arcs. By Theorem 8 we know that $K_{4}^{+}$admits $3 ! \cdot 4=24$ uniform factorizations. We also know that the automorphism group of $K_{4}^{+}$is Aut $K_{4}^{+}=\mathcal{S}_{4}$, and this enables us to compute the different equivalence classes of uniform factorizations of $K_{4}^{+}$by its automorphism group. These computations have been performed with the help of GAP (for details about GAP, see [10]). We summarize the results in Table 1.

From Table 1 we see that there are six equivalence classes of uniform factorizations of $K_{4}^{+}$. Representatives of three of them can be found between factorizations arising from any of the Latin squares which represent a composition table for $\mathbb{Z}_{4}$, and the other three arise only from the Latin square which is a composition table for $V_{4}$. More precisely, the permutation groups of uniform factorizations arising from the Latin square which is a 
Table 1. Uniform factorizations of $K_{4}^{+}$.

\begin{tabular}{lcccc}
\hline$G=G\left(K_{4}^{+}, F\right)$ & $|G|$ & Number factorizations & Equivalence classes & $\operatorname{Aut}\left(K_{4}^{+}, F\right)$ \\
\hline$V_{4}=\mathbb{Z}_{2}^{2}$ & 4 & 1 & 1 & $\mathcal{S}_{4}$ \\
$\mathbb{Z}_{4}$ & 4 & 3 & 1 & $D(8)$ \\
$D(8)$ & 8 & 6 & 2 & $D(8)$ \\
$\mathcal{A}_{4}$ & 12 & 2 & 1 & $\mathcal{A}_{4}$ \\
$\mathcal{S}_{4}$ & 24 & 12 & 1 & $\mathbb{Z}_{2}$ \\
\hline
\end{tabular}

composition table for $V_{4}$ are $V_{4}, D(8)$ and $\mathcal{A}_{4}$, and from any of the other three Latin squares we obtain $\mathbb{Z}_{4}, D(8)$ and $\mathcal{S}_{4}$. This is a consequence of Theorem 10 , to be proved in the next section, stating that uniform factorizations of a complete digraph with loops arising from isomorphic Latin squares are equivalent.

Case $r=5$. There are six uniform normalized Latin squares of order 5 (which are a composition table for $\left.\mathbb{Z}_{5}\right)^{2}$ :

$$
\begin{aligned}
& \left(\begin{array}{lllll}
1 & 2 & 3 & 4 & 5 \\
2 & 3 & 4 & 5 & 1 \\
3 & 4 & 5 & 1 & 2 \\
4 & 5 & 1 & 2 & 3 \\
5 & 1 & 2 & 3 & 4
\end{array}\right), \quad\left(\begin{array}{lllll}
1 & 2 & 3 & 4 & 5 \\
2 & 3 & 5 & 1 & 4 \\
3 & 5 & 4 & 2 & 1 \\
4 & 1 & 2 & 5 & 3 \\
5 & 4 & 1 & 3 & 2
\end{array}\right), \quad\left(\begin{array}{lllll}
1 & 2 & 3 & 4 & 5 \\
2 & 4 & 1 & 5 & 3 \\
3 & 1 & 5 & 2 & 4 \\
4 & 5 & 2 & 3 & 1 \\
5 & 3 & 4 & 1 & 2
\end{array}\right) \\
& \left(\begin{array}{lllll}
1 & 2 & 3 & 4 & 5 \\
2 & 4 & 5 & 3 & 1 \\
3 & 5 & 2 & 1 & 4 \\
4 & 3 & 1 & 5 & 2 \\
5 & 1 & 4 & 2 & 3
\end{array}\right), \quad\left(\begin{array}{lllll}
1 & 2 & 3 & 4 & 5 \\
2 & 5 & 1 & 3 & 4 \\
3 & 1 & 4 & 5 & 2 \\
4 & 3 & 5 & 2 & 1 \\
5 & 4 & 2 & 1 & 3
\end{array}\right), \quad\left(\begin{array}{lllll}
1 & 2 & 3 & 4 & 5 \\
2 & 5 & 4 & 1 & 3 \\
3 & 4 & 2 & 5 & 1 \\
4 & 1 & 5 & 3 & 2 \\
5 & 3 & 1 & 2 & 4
\end{array}\right)
\end{aligned}
$$

Let us apply this result again to a complete digraph with loops, $K_{5}^{+}$. By Theorem 8 , the digraph $K_{5}^{+}$admits $(r-1) ! 6=24 \cdot 6=144$ uniform factorizations. We compute them with GAP and show the resulting permutation groups in Table 2. Since Aut $K_{5}^{+}=\mathcal{S}_{5}$, we can classify the uniform factorizations of $K_{5}^{+}$in equivalence classes by the automorphism group. It transpires that there are six different equivalence classes and we can obtain representatives of all the six from factorizations arising from any of the Latin squares (by Theorem 10).

\section{Representation of permutation groups of uniform factorizations of line digraphs}

This section is devoted to the representation of permutation groups of small degree as permutation groups of uniform factorizations of regular connected line digraphs. 
Table 2. Uniform factorizations of $K_{5}^{+}$.

\begin{tabular}{lrccc}
\hline$G=G\left(K_{5}^{+}, F\right)$ & $|G|$ & Number factorizations & Equivalence classes & $\operatorname{Aut}\left(K_{5}^{+}, F\right)$ \\
\hline $\mathbb{Z}_{5}$ & 5 & 6 & 1 & $\mathbb{Z}_{5} \rtimes \mathbb{Z}_{4}$ \\
$\mathbb{Z}_{5} \rtimes \mathbb{Z}_{2}$ & 10 & 6 & 1 & $\mathbb{Z}_{5} \rtimes \mathbb{Z}_{4}$ \\
$\mathbb{Z}_{5} \rtimes \mathbb{Z}_{4}$ & 20 & 12 & 2 & $\mathbb{Z}_{5} \rtimes \mathbb{Z}_{4}$ \\
$\mathcal{A}_{5}$ & 60 & 60 & 1 & $\mathbb{Z}_{2}$ \\
$\mathcal{S}_{5}$ & 120 & 60 & 1 & $\mathbb{Z}_{2}$ \\
\hline
\end{tabular}

The importance of this study comes from the following fact. If a permutation group $G$ is the permutation group of a uniform factorization $F$ of a regular line digraph, then the Cayley cover of this digraph, $\operatorname{Cay}(G, F)$, is also a line digraph. Then, since the digraph $\operatorname{Cay}(G, F)$ is $G$-vertex-regular, the digraph $\mathrm{L}^{-1} \operatorname{Cay}(G, F)$ is $G$-arc-regular.

For example, let us consider the trivial case of regular connected line digraphs of degree 1, which are directed cycles. A cycle $C_{m}$ of order $m$ admits a unique factorization $F$, whose permutation group is the cyclic group $\mathbb{Z}_{m}$. Then, the corresponding Cayley cover is $\left(\bar{C}_{m}\right)_{F}=\operatorname{Cay}\left(\mathbb{Z}_{m}, F\right)=C_{m}$ and in particular, a line digraph. That is to say, the unique factorization that $C_{m}$ admits is uniform. Therefore, the cyclic groups $\mathbb{Z}_{m}$ represent all permutation groups of uniform factorizations of a regular line digraph of order $m$ and degree 1 . Thus, we only need to consider regular digraphs of degree $r>1$.

In general, let $\Gamma=(V, A)$ be a (connected) $r$-regular line digraph $\Gamma$. Since $\Gamma$ is assumed connected, the permutation group $G=G(\Gamma, F)$ of a factorization $F=\left\{f_{1}, \ldots, f_{r}\right\}$ acts transitively on $V$. Moreover, $F$ is a set of discordant permutations since a line digraph is never a multidigraph. Recall that $F$ is uniform if the Cayley cover $\bar{\Gamma}_{F}=\operatorname{Cay}(G, F)$ is a line digraph. By Theorem $1, \bar{\Gamma}_{F}$ is a line digraph if and only if $F F^{-1}$ is a subgroup of $G$ of order $r$. In such a case, $\bar{\Gamma}_{F}$ is $G$-vertex-regular and $\mathrm{L}^{-1}\left(\bar{\Gamma}_{F}\right)$ is $G$-arc-regular. Conversely, let $G$ be a permutation group which acts transitively on a set $V$. Then, $G$ is a permutation group of a uniform factorization of a $r$-regular line digraph $\Gamma=(V, A)$ if and only if $G$ is generated by a subset $F \subseteq G$ of $r$ pairwise discordant permutations of $V$, and the $F F^{-1}$ is a subgroup of $G$ of order $r$. Note that in this case the set $F$ is a right coset of $F F^{-1}$ in $G$.

The following characterization of arc-regular permutation groups is now straightforward to check:

Theorem 9 Let $G$ be a permutation group that acts transitively on a set $\Omega$ of $n$ elements. There exists a connected $r$-regular digraph $\Gamma$ of order $n / r$ that is $G$-arc-regular if and only if $G$ is generated by a subset $F \subseteq G$ of $r$ discordant permutations of $\Omega$ such that $F F^{-1}$ is a subgroup of $G$ of order $r$.

A simpler representation problem consists of determining which transitive groups of degree $r$ are actually permutation groups of uniform factorizations of the complete digraph with loops $K_{r}^{+}$(the only $r$-regular digraph of order $r$.) We can give a new characterization of such groups. It follows from the next theorem. 
Theorem 10 Let $\mathcal{Q}$ and $\mathcal{Q}^{\prime}$ be normalized uniform Latin squares of order r representing composition tables for isomorphic groups.

Then, permutation groups generated by uniform factorizations of $K_{r}^{+}$arising from $\mathcal{Q}$ are isomorphic to permutation groups of uniform factorizations $K_{r}^{+}$arising from $\mathcal{Q}^{\prime}$.

Proof: Let us denote the vertex set of the digraph $K_{r}^{+}$by $\{1, \ldots, r\}$, and let $\left\{\delta_{1}, \ldots, \delta_{r}\right\}$ and $\left\{\delta_{1}^{\prime}, \ldots, \delta_{r}^{\prime}\right\}$ be the complete sets of discordant permutations of $\mathcal{Q}$ and $\mathcal{Q}^{\prime}$, respectively. By Corollary 6, the Latin squares $\mathcal{Q}$ and $\mathcal{Q}^{\prime}$ are composition tables for the group $H$ generated by $\left\{\delta_{1}, \ldots, \delta_{r}\right\}$ (or $\left\{\delta_{1}^{\prime}, \ldots, \delta_{r}^{\prime}\right\}$.)

Let $F$ be the factorization of $K_{r}^{+}$defined by $\mathcal{Q}$, that is, in which an $\operatorname{arc}(j, k) \in$ $A\left(K_{r}^{+}\right)$is colored by $f_{i}$ if and only if $L(j, k)=i$, or equivalently, if and only if $i=k^{\delta_{j}}$. Then, $F=\left\{f_{1}, \ldots, f_{r}\right\}$ where $j^{f_{i}}=i^{\delta_{j}^{-1}}$ for $j \in V\left(K_{r}^{+}\right)$and $1 \leq i \leq r$. Analogously, let $F^{\prime}=\left\{f_{1}^{\prime}, \ldots, f_{r}^{\prime}\right\}$ be the factorization of $K_{r}^{+}$where $j^{f_{i}^{\prime}}=i^{\delta_{j}^{-1}}$ for $\bar{j} \in V\left(K_{r}^{+}\right)$and $1 \leq$ $i \leq r$.

We claim that the factorizations $F$ and $F^{\prime}$ of $K_{r}^{+}$are equivalent by an automorphism of $K_{r}^{+}$. By Corollary 6, we have that the Latin squares $\mathcal{Q}$ and $\mathcal{Q}^{\prime}$ are isomorphic. That is, there exist bijections $\sigma: R \rightarrow R^{\prime}, \tau: C \rightarrow C^{\prime}$, and $\pi: S \rightarrow S^{\prime}$, such that $L^{\prime}(i, j)=$ $L\left(i^{\sigma^{-1}}, j^{\tau^{-1}}\right)^{\pi}$ for $(i, j) \in R^{\prime} \times S^{\prime}$.

Without loss of generality we can assume that $\sigma=\pi:\{1, \ldots, r\} \rightarrow\{1, \ldots, r\}$. Indeed, we have that $(R, C, S ; L)$ and $\left(R^{\prime}, C^{\prime}, S^{\prime} ; L^{\prime}\right)$ are normalized and $L^{\prime}(i, j)^{\pi^{-1}}=L\left(i^{\sigma^{-1}}, j^{\tau^{-1}}\right)$. Then, let us consider the Latin square given by $L^{\prime}(i, j)^{\pi^{-1}}$. In order that the symbols in its first column be in natural order, it must be verified that $i^{\sigma^{-1}}=i^{\pi^{-1}}$ for $1 \leq i \leq r$.

Let us define the mapping $\phi: V\left(K_{r}^{+}\right) \rightarrow V\left(K_{r}^{+}\right)$by $i^{\phi}=i^{\sigma}$ for $1 \leq i \leq r$. Clearly, $\phi$ is an automorphism of $K_{r}^{+}$, and

$$
\begin{aligned}
\left(j^{\delta_{k}}\right)^{\phi} & =\left(j^{\delta_{k}}\right)^{\sigma}=L(k, j)^{\sigma}=L(k, j)^{\pi}=L\left(\left(k^{\sigma}\right)^{\sigma^{-1}},\right. \\
\left.\left(j^{\tau}\right)^{\tau^{-1}}\right)^{\pi} & =L^{\prime}\left(k^{\sigma}, j^{\tau}\right)=\left(j^{\tau}\right)^{\delta_{k^{\sigma}}} .
\end{aligned}
$$

Hence, $F$ and $F^{\prime}$ are equivalent by the automorphism $\phi$, as claimed.

Finally, since factorizations of $K_{r}^{+}$arising from an arbitrary Latin square $M$ of order $r$ coincide with factorizations of $K_{r}^{+}$defined by the Latin square $\bar{M}$ which is obtained by permuting the rows of $M$, it follows that factorizations of $K_{r}^{+}$arising from $\mathcal{Q}$ are equivalent to factorizations of $K_{r}^{+}$arising from $\mathcal{Q}^{\prime}$.

Thus, we do not need to calculate all permutation groups of uniform factorizations of $K_{r}^{+}$ arising from all uniform normalized Latin squares of order $r$. If $c$ is the number of pairwise non-isomorphic groups of given order $r$, it suffices to consider the $c$ Latin squares being composition tables for $c$ pairwise non-isomorphic representatives.

We apply these remarks to the case of transitive groups of degree $n$, where $n \leq 7$. It is proved in [8] that the only permutationally nonequivalent transitive groups of degree equal or less than seven are those listed in the first columns of Tables 3-6. We follow the same notation for them as in [8].

If a transitive group of degree $n$ is a permutation group of a uniform factorization of a $r$-regular (connected) line digraph of order $n$, then the degree of the digraph is necessarily 
Table 3. Transitive groups of degree 4.

\begin{tabular}{lcccc}
\hline & Order & Description & Digraph degree & Multidigraph degree \\
\hline $\mathrm{T} 4.1$ & 4 & $\mathbb{Z}_{4}$ & 1 & 2,4 \\
$\mathrm{~T} 4.2$ & 4 & $\mathbb{Z}_{2}^{2}$ & & 2,4 \\
$\mathrm{~T} 4.3$ & 8 & $D(8) \simeq \mathbb{Z}_{2} 2 \mathbb{Z}_{2}$ & 2 & 4 \\
$\mathrm{~T} 4.4$ & 12 & $\mathcal{A}_{4}$ & 2 & 4 \\
$\mathrm{~T} 4.5$ & 24 & $\mathcal{S}_{4}$ & 4 & \\
\hline
\end{tabular}

Table 4. Transitive groups of degree 5.

\begin{tabular}{lrccc}
\hline & Order & Description & Digraph degree & Multidigraph degree \\
\hline T5.1 & 5 & $\mathbb{Z}_{5}$ & 1 & 5 \\
T5.2 & 10 & $A S L_{1}(5) \simeq D(10)$ & & 5 \\
T5.3 & 20 & $A G L_{1}(5) \simeq \mathbb{Z}_{5} \rtimes \mathbb{Z}_{4}$ & & 5 \\
T5.4 & 60 & $\mathcal{A}_{5}$ & 5 & \\
T5.5 & 120 & $\mathcal{S}_{5}$ & 5 & \\
\hline
\end{tabular}

Table 5. Transitive groups of degree 6.

\begin{tabular}{|c|c|c|c|c|}
\hline & Order & Description & Digraph degree & Multidigraph degree \\
\hline T6.1 & 6 & $\mathbb{Z}_{6}$ & 1 & $2,3,6$ \\
\hline T6.2 & 6 & $\mathcal{S}_{3}$ & 2 & 3,6 \\
\hline T6.3 & 12 & $D(12)$ & 2 & 6 \\
\hline T6.7 & 12 & $\mathcal{A}_{6} \cap \mathrm{T} 6.6 \simeq \mathcal{A}_{4}$ & 3 & \\
\hline T6.12 & 18 & $\mathbb{Z}_{3} \imath \mathbb{Z}_{2}$ & 2 & 3,6 \\
\hline T6.5 & 24 & $\mathcal{A}_{6} \cap \mathrm{T} 6.4$ & 3 & \\
\hline T6.6 & 24 & $\mathcal{S}_{4}$ & 2,3 & 6 \\
\hline T6.8 & 24 & $\mathbb{Z}_{2} \imath \mathbb{Z}_{3}$ & 3 & 6 \\
\hline T6.10 & 36 & $\mathcal{A}_{6} \cap \mathrm{T} 6.9$ & 3 & \\
\hline T6.11 & 36 & $\mathbb{Z}_{3}^{2} \rtimes \mathbb{Z}_{2}^{2}$ & & 6 \\
\hline T6.4 & 48 & $\mathcal{S}_{2}$ 乙 $\mathcal{S}_{3}$ & 2 & 6 \\
\hline T6.14 & 60 & $P S L_{2}(5)$ & 3 & \\
\hline T6.9 & 72 & $\mathcal{S}_{3} \prec \mathbb{Z}_{2}$ & 2,6 & \\
\hline T6.13 & 120 & $P G L_{2}(5)$ & $2,3,6$ & 6 \\
\hline T6.15 & 360 & $\mathcal{A}_{6}$ & 3 & \\
\hline T6.16 & 720 & $\mathcal{S}_{6}$ & $2,3,6$ & 6 \\
\hline
\end{tabular}


Table 6. Transitive groups of degree 7.

\begin{tabular}{lrccc}
\hline & Order & Description & Digraph degree & Multidigraph degree \\
\hline $\mathrm{T} 7.1$ & 7 & $\mathbb{Z}_{7}$ & 1 & 7 \\
$\mathrm{~T} 7.2$ & 14 & $D(14)$ & & 7 \\
$\mathrm{~T} 7.3$ & 21 & $A S L_{1}(7)$ & & 7 \\
$\mathrm{~T} 7.4$ & 42 & $A G L_{1}(7)$ & & 7 \\
T7.5 & 168 & $P G L_{3}(2)$ & 7 & \\
T7.6 & 2520 & $\mathcal{A}_{7}$ & 7 & \\
T7.7 & 5040 & $\mathcal{S}_{7}$ & 7 & \\
\hline
\end{tabular}

a divisor of its order. For $n \leq 7$, it is easy to find all regular line digraphs of order $n$, and a procedure to calculate all the factorizations of a regular line digraph was given in the proof of Theorem 8 . Thus, we can manufacture all uniform factorizations of line digraphs of small degree and order, and calculate the corresponding permutation groups. We summarize the results (obtained with GAP) in Tables 3-6 at the end of the paper. These tables show the integers $r$ for which a transitive group of degree $n$ is an arc-regular permutation group of some regular (multi)digraph of degree $r$. Note that every transitive group of degree $n \leq 7$ represents an arc-regular permutation group of some (multi)digraph for some degree, but not every transitive group of degree $n \leq 7$ represents an arc-regular permutation group of some digraph (with no multiple arcs).

\section{Acknowledgments}

I am very grateful to Prof. Oriol Serra for his useful comments during the first writing of this manuscript. I also would very much thank the referees for their helpful remarks and for pointing out several inaccuracies in the original version.

\section{Notes}

1. These are all the normalized Latin squares of order $r=4$, but the number of normalized Latin squares of order $r$ (not necessarily uniform) grows quickly with $r$. For instance, for $r=5$ the number of normalized Latin squares is 56, for $r=6$ is 9408 and for $r=7$ is 16942080 (see [9].)

2. It is not difficult to check that there are 80 uniform normalized Latin squares of order 6 .

\section{References}

1. L. Babai, "Automorphism groups, isomorphism, reconstruction," in Handbook of Combinatorics, R. Graham, M. Grötschel, and L. Lovász (Eds.), Vol. 1-2, Elsevier, Amsterdam, 1995.

2. L. Babai, P.J. Cameron, M. Deza, and N.M. Singhi, "On sharply edge-transitive permutation groups," J. Algebra 73 (1981), 573-585.

3. R. Bailey, "Latin squares with highly transitive automorphism groups," J. Austral. Math. Soc. Ser. A 33 (1982), $18-22$. 
4. J.M. Brunat, Contribució a l'estudi de la simetria de grafs dirigits i les seves aplicacions, $\mathrm{PhD}$ thesis, Universitat Politècnica de Catalunya, Barcelona, 1994.

5. P.J. Cameron, "Digraphs admitting sharply edge-transitive automorphism groups," European J. Combin. 8 (1987), 357-365

6. A. Cayley, "On Latin squares," Messenger of Math. European 19 (1890), 135-137.

7. G. Chartrand and L. Lesniak, Graphs \& Digraphs, 3rd. edition, Chapman \& Hall, London, 1996.

8. J.D. Dixon and B. Mortimer, Permutation groups, Springer Verlag, New York, 1996.

9. M.J. Erickson, Introduction to Combinatorics, John Wiley \& Sons, Inc., New York, 1996.

10. The GAP Group, GAP_Groups, Algorithms, and Programming, Version 4.2, Aachen, St Andrews, 1999 , (http://www-gap.dcs.st-and.ac.uk/ gap).

11. R.L. Hemminger and L.W. Beineke, "Line graphs and line digraphs," in Selected Topics in Graph Theory I, L.W. Beineke and R.J. Wilson (Eds.), Academic Press, London, 1978.

12. S.P. Mansilla and O. Serra, "Construction of $k$-arc transitive digraphs," Discrete Math. to appear.

13. S.P. Mansilla and O. Serra, "Automorphism groups of $k$-arc transitive covers," Discrete Math. submitted.

14. J. Lützen, G. Sabidussi, and B. Toft, "Julius Petersen 1839-1910: A biography," Discrete Math. 100 (1992), 9-82.

15. J.H. van Lint and R.M. Wilson, A Course in Combinatorics, Cambridge University Press, Cambridge, 1992. 